\title{
From Superdeformations to Hyperdeformations
}

P. Fallon ${ }^{\mathrm{a}}$

${ }^{a}$ Nuclear Science Division, Lawrence Berkeley National Laboratory, 1, Cyclotron Road, Berkeley CA 94720

\section{INTRODUCTION}

It is possible that some nuclei may attain even greater quadrupole deformations than those observed in superdeformed nuclei and the search for such hyperdeformed shapes has been a goal of nuclear structure for many years.

In this talk I will discuss some recent developments in the experimental study of superdeformed nuclei and in particular the search for the more exotic hyperdeformed nuclei. I'll begin by briefly reviewing the basic concepts connected with nuclei at extreme deformations and then turn to recent attempts to extend these studies from super to hyperdeformations, with the focus being on our work in the cadmium and tin region. Finally, I will say a few words on longer term future prospects.

First observed in ${ }^{242} \mathrm{Am}$ [1], through the discovery of fission isomerism and then understood a few years later as a second minimum in the fission barrier, superdeformation is now established in several nuclear mass regions ranging from $A \sim 40$ through $A \sim 240$. Following the observation of fission isomers there was a gap of about 25 years before the first high-spin superdeformed nucleus was discovered [2]: a wait determined by the need for multi detector high-resolution gamma-ray detector arrays. To date we have observed and studied over 300 superdeformed rotational bands throughout the nuclear chart [3]. A review of the physics of superdeformed nuclei and references can be found in [4].

The existence of superdeformation is closely coupled to the concept of deformed shell gaps (secondary minima) and is a clear illustration of the role of single-particle (microscopic) effects on macroscopic properties. The spectrum of single-particle states derived from an axially-symmetric harmonic oscillator potential provides the simplest realization of superdeformed magic shell gaps [5]. Here, degeneracies (symmetries) in the singleparticle spectrum occur when the deformation corresponds to an ellipsoid with integer values for the major-to-minor axis ratios; e.g. spherical (1:1 axis ratio), superdeformed $(2: 1)$ or hyperdeformed $(3: 1)$ and they generate the shell gaps that help stabilize the nuclear deformation.

An important point is that shell gaps arising from shape symmetries occur at a deformation that is independent of nuclear mass; e.g. for the harmonic oscillator all superdeformed nuclei will have a prolate $2: 1$ axis ratio that corresponds to a quadrupole deformation of $\beta_{2} \approx 0.65$. Superdeformed nuclei can thus be considered to be the deformed analog of spherical magic nuclei and are a phenomenon distinct from normal deformations. (The 
strict independece of deformed shells and mass is valid in the harmonic oscillator potention. I will discuss the impact of more realsitic potential later.)

Figure 1 illustrates the distinction between normal and superdeformations further. Here I plot the normalized quadrupole moment, which is proportional to the quadrupole deformation obtained from $\mathrm{B}(\mathrm{E} 2)$ values, for nuclei in the mass range $A \approx 10-200$ (data are from [6]). The deformation, expressed in terms of axis ratios, is given to the right side. Bohr and Mottelson provide [7] a simple estimate for the nuclear deformation in the absence of shell corrections, ie. for a more-or-less uniform level-energy spectrum. They show that the deformation $\delta$ is proportional to $1 / A^{1 / 3}$ (full line); a behavior that can be thought of as arising from the competing effects of the core nucleons (volume effect $\propto A$ ) and valence nucleons (surface effect $\propto A^{2 / 3}$ ) that provide the spherical and deformation driving tendencies, respectively. In figure 1 we see that the ground state deformations of nuclei away from closed shells follow this $1 / A^{1 / 3}$ dependence, but there strong deviations in the vicinity of closed spherical shells (e.g. $\left.{ }^{208} \mathrm{~Pb}\right)$.

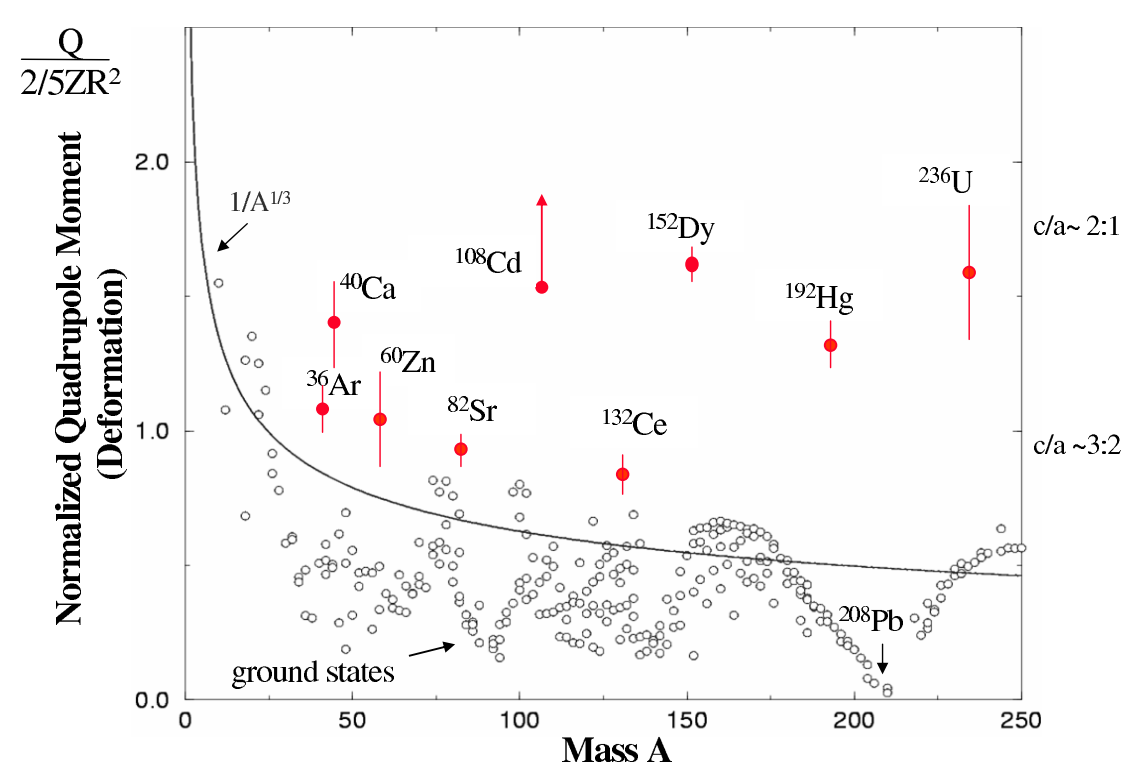

Figure 1. Measured quadrupole moments scaled to remove the $Z$ dependence for both normal deformed (open circles), and superdeformed (filled circles) nuclei. The The solid line represents the $A^{-1 / 3}$ dependence discussed in the text. Data on normal deformed nuclei were taken from [6]. Superdeformed data are from [3] or references provided in the text. The approximate values of the scaled quadrupole moment for prolate deformations with major:minor axis ratios of 3:2 and 2:1 are indicated on the right.

The experimentally derived deformations obtained from normalized quadrupole mo- 
ments for selected superdeformed nuclei are also shown in fig. 1. For nuclei $\mathrm{A} \sim 150$, 190, and 240 there are large deviations from the $1 / A^{1 / 3}$ trend due to the superdeformed shell gaps. These deviations are comparable to the effects of the spherical shell gaps at $\mathrm{Z}=82, \mathrm{~N}=126\left({ }^{208} \mathrm{~Pb}\right)$, but in the opposite direction. The superdeformed nuclei in these three regions are clearly separated from their normal deformed counterparts. They are restricted to distinct mass regions with deformation values that are rather close to each other; thus supporting the concept of deformed magic shell closures that occur because of nuclear shape symmetries. Below $A \sim 150$ two effects combine to blur the distinction between superdeformed and normal deformed nuclei. First, in very light nuclei even "normal" deformations driven by the polarizing tendencies of the valence nucleons can be large with magnitudes approaching the superdeformed limit. Second, measured deformations of most superdeformed nuclei deviate significantly from the ideal 2:1 shape and in some cases form continuous chains of deformations spanning the range from normal deformed to superdeformed (as shown, for example, in the figure on page 12 in ref. [3]).

Although the picture of deformed magic shell gaps presented by the harmonic oscillator is oversimplified, the notion of superdeformation as a phenomenon closely connected with the occurrence of deformed (magic) shell gaps imparts a useful basis within which to examine and characterize their properties. Superdeformation is a phenomenon whose origin goes beyond the deformation-driving tendencies of the valence nucleons $\left(\sim 1 / A^{1 / 3}\right)$, and is one that provides a beautiful illustration of the interplay between the microscopic and macroscopic degrees of freedom.

\subsection{The Intruder Classification Scheme}

In the simple limit based on a harmonic oscillator potential superdeformed nuclei have a deformation corresponding to a prolate axis ratio $\mathrm{c} / \mathrm{a}=2$ and hyperdeformed nuclei have $\mathrm{c} / \mathrm{a}=3$. However, realistic nuclear potentials modify this simple picture and superdeformed nuclei are observed with axis ratios in the range $\mathrm{c} / \mathrm{a} \sim 1.6$ to 1.8. Similarly we may expect hyperdeformed nuclei to exhibit c/a values smaller than 3 , and classifying the structure according the precise value of the nuclear deformation may not be so useful. It turns out, nevertheless, that each distinct deformed shape (whether normal-, super-, or hyperdeformed) can generally be correlated with the occupation of specific high-j intruder states [8]. Normal deformed nuclei correspond to the occupation of intruders from one major oscillator shell above the spherical Fermi surface (N+1,"normal-intruders"), superdeformed nuclei can be correlated with the occupation of intruder states from two shells above $(\mathrm{N}+2$, "super-intruders"), and in analogy we may expect hyperdeformed nuclei to involve intruder states from three shells above ( $\mathrm{N}+3$, "hyper-intruders").

This correspondence between deformation and intruder occupation is nicely illustrated by the evolution of the proton $i_{13 / 2}$ state with deformation and Z. It starts out at the Fermi surface for spherical nuclei around $\mathrm{Z}=100$ and for normal deformed $\left(\beta_{2} \sim 0.2\right)$ nuclei around $\mathrm{Z}=92$, and in these regions the $\pi i_{13 / 2}$ state is the normal-intruder state lowered by one oscillator shell due to the spin-orbit interaction. High-j states have large quadrupole moments and they are lowered even further by increasing the quadrupole deformation until at superdeformations $\left(\beta_{2} \sim 0.6\right)$ the $i_{13 / 2}$ state is at the Fermi surface for $\mathrm{Z} \sim 66$ (e.g. $\left.{ }^{152} \mathrm{Dy}\right)$. At this point the $i_{13 / 2}$ intruder has dropped two major oscillator shells and it can be classified as a super-intruder. By the time we get to Cadmium, $Z=48$, the $i_{13 / 2}$ state 
has come down 3 major shells, and it seems natural to label this orbital a hyper-intruder.

In section 2 the intruder classification is used scheme to characterize the nuclear structure (rather than the deformation value) of very deformed rotational bands in Cd nuclei and also as a guide to experimental searches for hyperdeformation.

\subsection{The Regions of Superdeformation}

With each region discovered different aspects of the physics of very deformed rotating nuclei have been emphasized; e.g. around A 150 the extreme single-particle properties were readily identified providing insight into the symmetries and extreme "single-particle" nature of superdeformed nuclei, while in the A 190 mass region the pairing correlations were seen to have a significant influence on the rotation of the superdeformed nucleus, promoting a deeper understanding of the importance of higher order pairing terms. The observation of a rich variety of superdeformed nuclei provided an ideal opportunity to study practically all aspects of nuclear structure: from shell structure (e.g., exotic states; extreme single-particle motion) to collective modes (e.g. rotations, pairing, vibrations).

Most of the $\sim 300$ superdeformed bands discovered correspond to excitations within the second minimum and the vast majority are single-particle excitations. There are a limited number of collective excitations (vibrations) concentrated in heavier systems (see [9] [10] and references therein). One remarkable and unexpecetd facet of superdeformed nuclei was the observation of identical rotational bands [11], which proved to be a very stringent test of theory that demanded highly accurate calulation of the deformation, pairing, and alignment properties.

Not all challenges have been theoretical. On the experimental front it has proven extremely difficult to observe the gamma-ray decays connecting the superdeformed to normal deformed states and by-and-large the spins, parities, and excitations of most supertdeformed bands have not been measured.

The status of the data on superdeformed to normal deformed links is summarized as follows (see [3] for references or individual citations); A $\sim 40$ (all linked $-{ }^{36} \mathrm{Ar},{ }^{40} \mathrm{Ca}$ ); A 60 ( $\sim 50 \%$ linked); A 80 ( $\left.{ }^{84} \mathrm{Zr}\right)[12] ; \mathrm{A} \sim 130$ ( $\sim 50 \%$ linked $) ; \mathrm{A} \sim 150\left({ }^{149} \mathrm{Gd},{ }^{152} \mathrm{Dy}\right) ; \mathrm{A} \sim 190$ $\left({ }^{194} \mathrm{Hg},{ }^{194} \mathrm{~Pb},{ }^{192} \mathrm{~Pb}[13]\right) ; \mathrm{A} \sim 240{ }^{236,238} \mathrm{U}$. The importance of a complete knowledge of the properties of superdeformed states is seen clearly in the case of ${ }^{36} \mathrm{Ar}$ [14], where a knowledge of spins parities, energies and B(E2)'s have enabled us to study the microscopic origin of deformations and rotational collectivity through a comparison with large scale spherical shell models. Observing the decays from the second minimum to the first also gives information on the tunneling process between two distinct minima and these data are needed to understand, for example, whether the decay is statistical, if there are structure effects, how the decay depends on excitation energy, and how it varies from one mass region to another.

I would like to end this introduction with the comment that experiments on superdeformed nuclei played an important role in advancing the theoretical models and tools used to describe collective nuclear phenomena, and many of these developments are now being applied in calulations in different mass regions, for all deformations, and in a variey of spin regimes. 


\section{FROM SUPER TO HYPERDEFORMATION}

Hyperdeformed nuclei exist in a region very close to the fission limit and there is reason to believe that the theories and models successfully used to predict the regions and properties of superdeformed nuclei may not be applicable to hyperdeformed nuclei. Their shape is expected to deviate significantly from a pure ellipsoid and "necking" degrees of freedom may play an important role. These factors affect how well one can calculate the macroscopic energy, which is critical for a detailed understanding of fission barriers and the shape of the potential energy surface. The large deformation and very high angular momentum will modify the microscopic structure by bringing together levels from multiple oscillator-shells and a key question is whether there are favored nucleon numbers (shell gaps) that stabilize these exotic deformations and what are the relevant single-particle states? In addition, the extra degree of freedom due to the formation of a neck (the nucleus has a dumb-bell shape) can lead to new effects and it is an open question how properties, such as pairing correlations, will be affected.

Many experiments have been performed to search for nuclear hyperdeformation, but to date none have shown convincing evidence for discrete hyperdeformed bands. Generally speaking the observation of very deformed shapes requires several factors; e.g., favorable macroscopic and microscopic properties and favorable experimental conditions (input spin, excitation energy), but the precise location of the islands of hyperdeformed nuclei and the experimental conditions needed to populate these exotic structures remain unknown.

Here I want to mention two experimental programs carried out by our collaboration. The first is centered on nuclei in the $\mathrm{A} \sim 100 \mathrm{Cd} / \mathrm{Sn}$ region; i.e., ${ }^{106,108} \mathrm{Cd}$ (produced using the reactions ${ }^{48} \mathrm{Ca}+{ }^{62,64} \mathrm{Ni}$ ) and ${ }^{112,114} \mathrm{Sn}$ (produced using the reaction ${ }^{48} \mathrm{Ca}+{ }^{68,70} \mathrm{Zn}$. The second is centered on the $\mathrm{A} \sim 120 \mathrm{Ba} / \mathrm{Xe}$ region; i.e., ${ }^{126} \mathrm{Ba}$ and ${ }^{126} \mathrm{Xe}$, produced in the ${ }^{64} \mathrm{Ni}+{ }^{64} \mathrm{Ni}$ and ${ }^{48} \mathrm{Ca}+{ }^{82} \mathrm{Se}$ reactions. I will focus on the first of these, the experiments on the $\mathrm{Xe}$ and $\mathrm{Ba}$ nuclei will be discussed in the contribution to these proceeding from B. Herskind et al. In both regions these nuclei can sustain the highest spins possible and can be produced by favorable (high spin-input and "cold") reactions.

\subsection{Hyper-intruder States in ${ }^{108} \mathrm{Cd}$ and the Search in Neighboring Cd and Sn Nuclei}

Following from the previous discussion of hyper-intruders we can speculate that a key element to observing hyperdeformed nuclei is to find examples where the $\mathrm{N}+3$ intruders can be populated. An N+3 intruder, for example, can be "brought" to the Fermi surface by either increasing the nucleon number and/or increasing the deformation. High angular momentum also favors the occupation of high-j, hyper-intruders states. Cadmium and Tin nuclei lie at the top of the $\mathrm{Z}=28-50$ shell, which means that the necessary $\mathrm{N}+3$ hyperintruders $\left(i_{13 / 2}\right.$ protons and $j_{15 / 2}$ neutrons in this case) approach the Fermi surface at a lower deformation than, say, a mid shell nucleus and may therefore be easier to populate. Recall that the A 190 superdeformed nuclei have a lower deformation than those around $\mathrm{A} \sim 150$ and both correspond to the occupation of $i_{13 / 2}$ protons and $j_{15 / 2}$ neutrons, whereas these particular super-intruders are not populated in bands observed in the lower portion of the shell (i.e. with $\mathrm{A} \sim 140$ ).

Calculations by Chasman [15] indicate that several nuclei near $\mathrm{A}=100$ (e.g. ${ }^{108} \mathrm{Cd}$, 
${ }^{110} \mathrm{Cd},{ }^{112} \mathrm{Sn},{ }^{114} \mathrm{Sn}$ ) have significant shell correction energies for deformed shapes with c/a $\sim 2.3$ and that these states are yrast around $\mathrm{I} \sim 62-65 \hbar$. Thus there is good reason to expect that these minima can be populated and the bands observed.

An experiment to search for very extended nuclear shapes in ${ }^{108} \mathrm{Cd}$ was performed on Gammasphere [16] at ATLAS using the reaction ${ }^{64} \mathrm{Ni}\left({ }^{48} \mathrm{Ca}, 4 \mathrm{n}\right)$ at $207 \mathrm{MeV}$. This resulted in the observation of two rotational bands [17] [18] at very high angular momentum. The bands, shown in figure 2, carry about $1.4 \%$ and $0.6 \%$ of the intensity of the ${ }^{108} \mathrm{Cd}$ channel, which carries approximately $15 \%$ of the total fusion-evaporation intensity. It is estimated (assuming the moments of inertia $\Im^{(1)}=\Im^{(2)}$ at the highest spins where is $\Im^{(2)}$ constant) that they extend over a spin range from about 40-60 $\hbar$. The bands have large dynamic moments of inertia, $\Im^{(2)}$, shown in the insert to figure 2, consistent with a very large prolate deformation of $\mathrm{c} / \mathrm{a} \sim 2$.
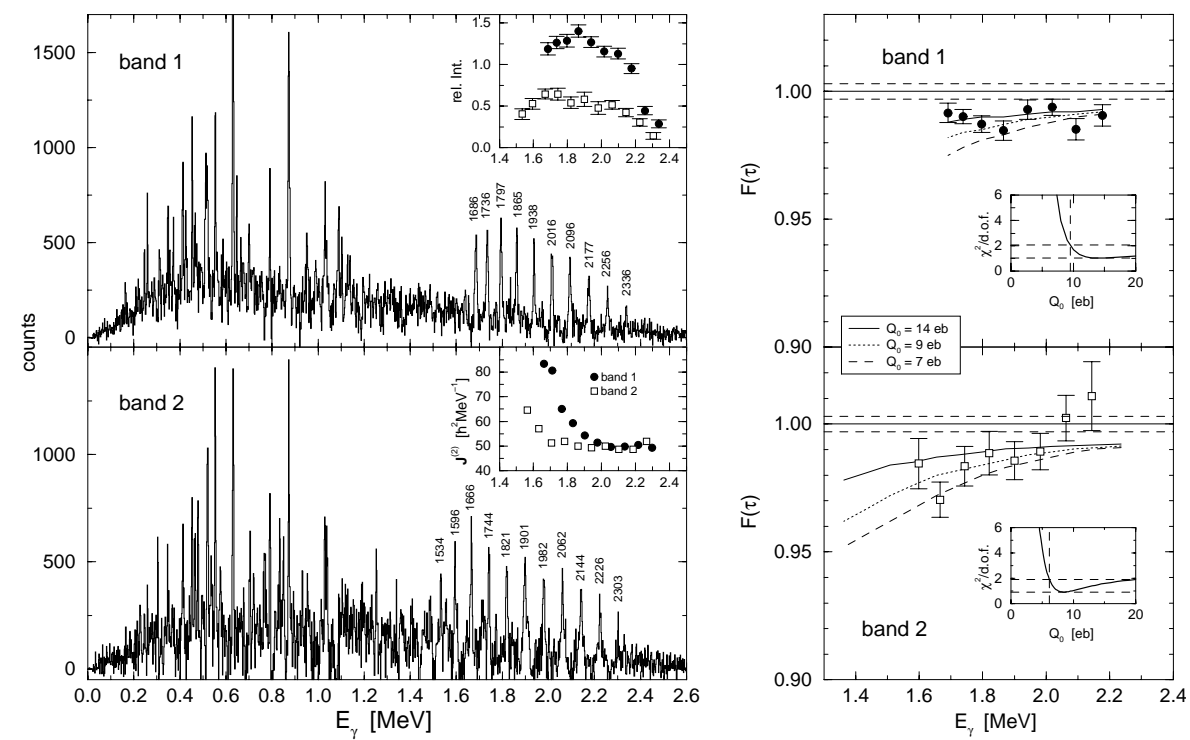

Figure 2. Spectra of bands 1 and 2 in ${ }^{108} \mathrm{Cd}$. The insets show the relative intensities of the bands with respect to the total intensity of the reaction channel (upper panel), and the dynamic moments of inertia (lower panel). The values for band 1 are given by the full circles, those for band 2 by the open squares. Right: Results of the fractional Doppler shift analysis. The lines show examples for calculated $\mathrm{F}(\tau)$ curves. The 2 fits for the calculated curves to the data as a function of the quadrupole moment $\mathrm{Q}_{0}$ are shown in the insets. The vertical dashed lines indicate the lower limit of the quadrupole moment consistent with a 1 error.

A measure of the transition state lifetimes (via the slowing down of the recoil in the 
target) confirmed their large deformation. Band 1 has a lower limit for the quadrupole moment of $Q_{t}=9.5 \mathrm{eb}(\mathrm{c} / \mathrm{a} \sim 1.8)$. Band 2 has a best-fit $Q_{t}=8.5 \mathrm{eb}(\mathrm{c} / \mathrm{a} \sim 1.7)$ and a lower limit of $6.2 \mathrm{eb}$. In both cases it was not possible to establish a meaningful upper limit.

The bands exhibit a rapid increase in $\left.\Im^{(2)}\right)$ at the lowest frequencies. Calculations [19] suggested that the rise in $\Im^{(2)}$ in band 1 coincides with the occupation of the proton $i_{13 / 2}$ hyper-intruder state. A recent study by Dudek (private communication) also places the $i_{13 / 2}$ proton at the Fermi surface for $\mathrm{Z} \sim 48$ and deformations of $\mathrm{c} / \mathrm{a} \sim 2$.

This evidence for the occupation of the proton hyper-intruder in ${ }^{108} \mathrm{Cd}$ suggests the exciting possibility that hyperdeformed nuclei (with both $i_{13 / 2} A$ proton and neutron $j_{15 / 2}$ hyper-intruders occupied) could be observed in this region. The $j_{15 / 2}$ hyper-intruder is predicted [20] to lie at the Fermi surface for $\mathrm{N}=64$, i.e. ${ }^{112} \mathrm{Cd}$ or ${ }^{114} \mathrm{Sn}$, at a deformation only slightly larger (10-20\%) than the ${ }^{108} \mathrm{Cd}$ bands and only 4 neutrons away. Moreover, this intruder could be occupied for $\mathrm{N}<64$ (e.g. ${ }^{110} \mathrm{Cd}_{62}$ or ${ }^{112} \mathrm{Sn}_{62}$ ) if the excitation energy is increased and/or the deformation increases.

Table 1

Comparison of reaction conditions for the Gammasphere "hyper-intruder" experiments.

\begin{tabular}{llll}
\hline Reaction & Beam Energy $(\mathrm{MeV})$ & $\mathrm{E}_{\text {compound }}(\mathrm{MeV})$ & $\mathrm{L}_{\text {max }} \hbar$ \\
\hline${ }^{64} \mathrm{Ni}\left({ }^{48} \mathrm{Ca}, 4 \mathrm{n}\right){ }^{108} \mathrm{Cd}$ & 207 & 97 & 80 \\
${ }^{62} \mathrm{Ni}\left({ }^{48} \mathrm{Ca}, 4 \mathrm{n}\right){ }^{106} \mathrm{Cd}$ & 207 & 96 & 77 \\
${ }^{68} \mathrm{Zn}\left({ }^{48} \mathrm{Ca}, 4 \mathrm{n}\right){ }^{112} \mathrm{Sn}$ & 206 & 96 & 78 \\
${ }^{70} \mathrm{Zn}\left({ }^{48} \mathrm{Ca}, 4 \mathrm{n}\right){ }^{114} \mathrm{Sn}$ & 202 & 97 & 80 \\
\hline
\end{tabular}

Following the observation of the hyper-intruder bands in ${ }^{108} \mathrm{Cd}$ and the favorable expections from calculations we carried out a series of very high statistics experiments to search for the analogous structures in ${ }^{106} \mathrm{Cd},{ }^{112} \mathrm{Sn}$ and ${ }^{114} \mathrm{Sn}$ using very similar reactions; e.g., ${ }^{48} \mathrm{Ca}$ on targets of ${ }^{62} \mathrm{Ni},{ }^{68} \mathrm{Zn}$, and ${ }^{70} \mathrm{Zn}$, respectively (table 1 ). In the latter two cases it was anticipated that the $j_{15 / 2}$ hyper-intruder neutron state may also be populated. However, no such hyper-intruder bands were observed in any of these additional experiments. This is a puzzle; from what we know about superdeformed shapes in all other mass regions we would have expected to find several nuclei around ${ }^{108} \mathrm{Cd}$ to exhibit hyper-intruder bands, especially since the ${ }^{108} \mathrm{Cd}$ structure is relatively strong $(\sim 1 \%$ of the 4n decay channel).

The non-observation of hyper-intruder bands in the ${ }^{106} \mathrm{Cd},{ }^{112} \mathrm{Sn}$, and ${ }^{114} \mathrm{Sn}$ experiments could be interpreted to mean that rotational states associated with $\mathrm{N}+3$ hyper-intruder levels in these nuclei are significantly less favored compared with ${ }^{108} \mathrm{Cd}$, this would be an important conclusion which impacts the direction of future searches for hyperdeformed states. However, we cannot rule out the possibilty that the population of hyper-intruder states depends sensitively on beam energy. Evidence for this is suggested by recent data on the unresolved gamma-ray continuum spectrum in ${ }^{126} \mathrm{Ba}$ [21]. These data show a ridge structure consistent with the decay of hyperdeformed states (similar ridges are also seen 
in the Xe data [22]). The surprising fact is that this ridge is seen in the ${ }^{64} \mathrm{Ni}+{ }^{64} \mathrm{Ni}$ reaction at $261 \mathrm{MeV}$, but no ridge is seen when the beam energy is lowered to $255 \mathrm{MeV}$. This corresponds to a $\sim 3 \mathrm{MeV}$ change in the center of mass energy and if confirmed would suggest a very strong dependence on beam energy.

\subsection{Future Prospects}

Present day high-statistics experiments performed with either Gammasphere or Euroball have reached a limit of $\sigma \sim 50 \mu$ barns for observing hyperdeformed states, and despite the high sensitivity and numerous attempts we have yet to obtain clear experimental evidence for hyperdeformation. While it may well turn out that todays instruments and facilities will provide the capability to observe hyperdeformation, significant improvements in the present level of sensitivity will require something new. Such developments in both the instrumentation (gamma-ray tracking) and heavy-ion beams (radioactive beams) are underway, and they will give orders of magnitude improvement in gamma-ray detection sensitivity as well as allow access to new nuclides.

\subsubsection{Radioactive Beams}

The topic of radioactive beams (RIBS) is usually discussed in connection with the isospin degree of freedom; however, it is very possible that the availability of neutron-rich RIBS will provide new capabilities to study exotic nuclear phenomena within the spin degree of freedom. To illustrate this consider again the " ${ }^{108} \mathrm{Cd}$ story" and the goal to populate the $j_{15 / 2}$ hyper-intruder. In this case even a few extra neutrons can make all the difference, and with a RIB beam of ${ }^{95} \mathrm{Kr}$ on a ${ }^{26} \mathrm{Mg}$ target it will be possible to produce ${ }^{121} \mathrm{Cd}$ as the compound system. Even allowing for the evaporation of 7 neutrons this still brings us to ${ }^{114} \mathrm{Cd}$, which is 6 neutrons beyond where we can go today (using a favorable "high-spin" reaction), and is a huge gain.

In addition, the more neutrons a given isotope has, then the more spin the nucleus can sustain. This will clearly favor the population of hyperdeformed states. Figure 3 shows the calculated maximum spin that a given isotope can sustain (for $\mathrm{Cd}$ to $\mathrm{Yb}$ ). The estimated maximum spin corresponds to the spin at which the fission barrier $\left(B_{f}\right)$ is equal to the neutron separation energy $\left(S_{n}\right)$, and was calculated using the analytic experssions of W.Swiatecki and W.D.Myers (seee the discussion in [23] for relevant formulae and references). In many cases the use of RIBS increases the available spin by up to $10 \hbar$. This is a significant increase and opens up a new spin regime for future study.

\subsubsection{Gamma-Ray Energy Tracking Detectors}

Just as Compton suppressed multidetector germanium arrays gave the experimental capability to discover and study superdeformed nuclei throughout the nuclear chart (as well as a host of other physics), the next generation of arrays based on a shell of highly segmented germanium detectors will provide orders of magnitude further increase in sensitivity and, amongst other things, give us the necessary tools to take the step from super to hyperdeformations. These new arrays will use gamma-ray energy tracking techniques [24] and represent the future benchmark for gamma-ray studies in almost all aspects of nuclear structure physics. The planned arrays, such as GRETA [25] in the USA and AGATA [26] in Europe, will be particularly important to capitalize on the new opportunities provided by future RIB facilties using both "fast-beam" (fragmentation) and "slow-beam" 


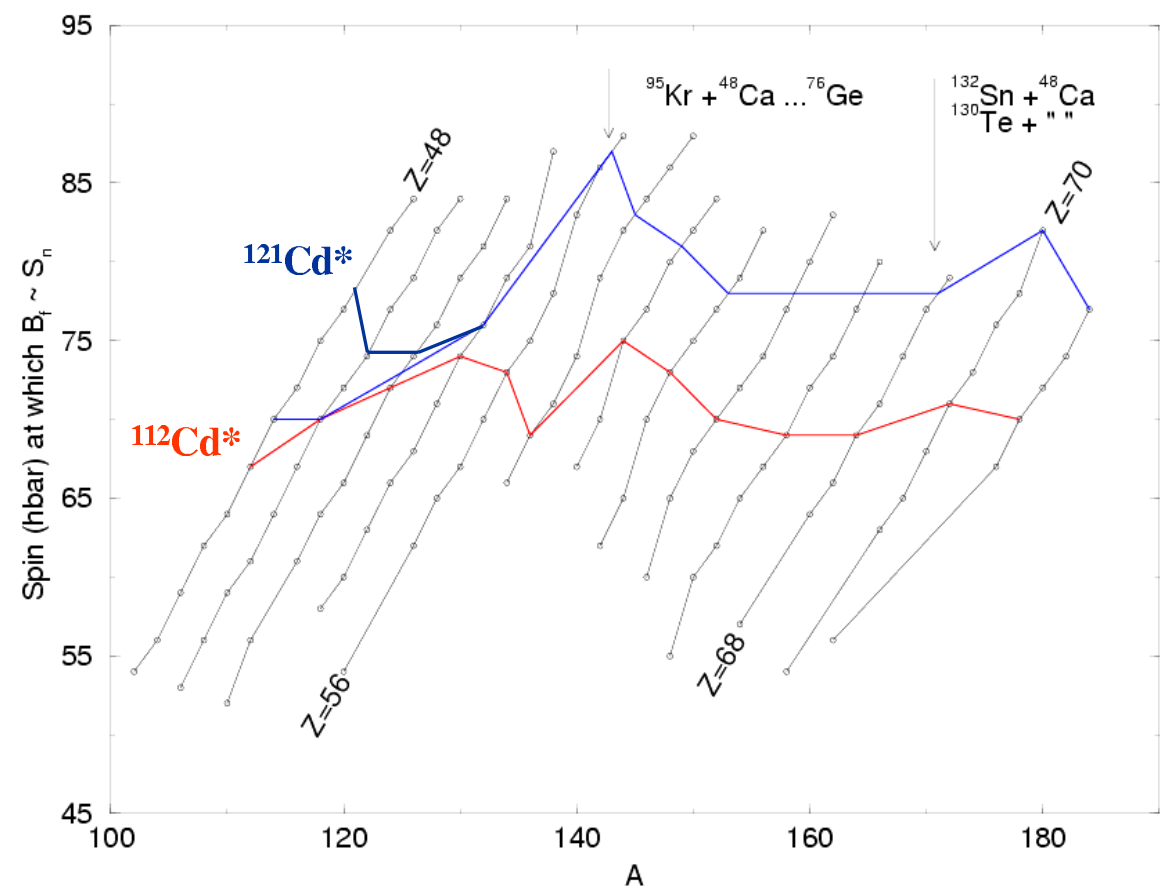

Figure 3. Calculated values (circles) for the angular momentum at which the fission barrier in a rotating Thomas Fermi model equals the neutron separation energy. This vales is taken as a measure of the max spin, $L_{\max }$ a nucleaus can sustain. The near verticle lines connect isotopes. The horizotal lines show the most neutron rich compound system accessible at very high spins by stable (lower) and radioactive (upper) beams.

(ISOL/re-accelerated) techniques. In the USA the first phase of GRETA is underway with the construction of GRETINA [27], which will have around 30 segemented coaxial gremanium crystals (depending on the final geometry). Each crystal has 36 electrical segments. The preferred design has four crystals enclosed in a common cryostat, and GRETINA is $1 / 4$ of the full $4 \pi$ array, GRETA.

\section{ACKNOWLEDGEMENTS}

The experiments to observe hyper-intruder bands in $\mathrm{Cd}$ and Sn nuclei were carried out by a collaboration involving many institutes and participants, worldwide; Lawrence Berkeley National Laboratory, Argonne National Laboratory, Niels Bohr Institute, University of Bonn, CEA Saclay, University of York, Florida State University. I would like to thank all my colleagues who contributed to this work and particularly B.Herskind (NBI) and H.Hubel (Bonn) who played a major role in planning the program of experiments 
discussed here as well as those I did not have time not discuss (e.g. in $\mathrm{Ba} / \mathrm{Xe} / \mathrm{Nd} / \mathrm{Yb}$ nuclei).

\section{REFERENCES}

1. S.M.Polikanov, et al., Soviet Phys. JETP 15 (1962) 1016; V.P. Pereligyn, et al., Soviet Phys. JETP 15, (1962) 1022.

2. P.J.Twin et el., Phys. Rev. Lett. 57 (1986) 81.

3. B.Singh, R.Zywina, R.B.Firestone, Table of Superdeformed Nuclear Bands and Fission Isomers, Third Edition, LBL-38004 (2002); Nucl.Data Sheets 97 (2002) 241.

4. D.Ward and P.Fallon, High Spin Properties of Atomic Nuclei, Advances in Nuclear Physics, Volume 26, Plenum Press New York (2001) p167.

5. A.Bohr and B.Mottelson, Nuclear Structure, Vol II, Benjamin, New York (1975) p592.

6. S.Raman et al., Atomic Data and Nucl. Data Tables, 36 (1987) 1.

7. A.Bohr and B.Mottelson, Nuclear Structure, Vol II, Benjamin, New York (1975) p49.

8. W.Nazarewicz, Contemporary Topics in Nuclear Structure Physics, World Scientific (1988) 467.

9. M.Hunyadi et al., Phys. Lett. B505 (2001) 27, and references therein.

10. T.Lauritsen et al., Phys. Rev. Lett. 89 (2002) 28250.

11. C.Baktash, B.Haas, and W.Nazarewicz, Ann. Rev. Nucl. Part. Sci 45 (1995) 485.

12. C.J.Chiara et al., International Conf. on The Limits of Nuclear Structure, AIP Conference Proceedings to be published.

13. A.N.Wilson et al., Phys. Rev. Lett. 90 (2003) 3142501.

14. C.E.Svenson et al., Phys. Rev. Lett. 85, (2000) 2693.

15. R.R.Chasman, Phys. Rev. C64 (2001) 024311.

16. I-Y.Lee, Nucl. Phys A520 (1990) 641c.

17. R.M.Clark et al., Phys. Rev. Lett. 87 (2001) 202502.

18. A.Goergen et al., Phys. Rev. C65 (2002) 027302.

19. C.T.Lee et al., Phys. Rev. C66 (2002) 041301(R).

20. Y.Sun, private communication.

21. B.Herskind et al., International Conf. on The Labyrinth in Nuclear Structure, AIP Conference Proceedings Volume 701 (2004) 303-313.

22. B.Herskind et al., contribution to these proceedings.

23. D.Ward et al., Phys. Rev. C66 (2002) 024317.

24. I-Y.Lee, Nucl. Instr. Meth A422 (1999) 195; M.A. Deleplanque et al., Nucl. Instr. Meth. A430 (1999) 292

25. I-Y.Lee, International Conf. on The Frontiers of Nuclear Structure, AIP Conference Proceedings Volume 656 (2005) 243-348, and references therein.

26. D.Bazzacco, International Conf. on The Labyrinth in Nuclear Structure, AIP Conference Proceedings Volume 701 (2004) 265-272, and references therein.

27. I-Y.Lee, International Conf. on The Limits of Nuclear Structure, AIP Conference Proceedings to be published. 